\title{
A Wideband Stacked Patch Antenna with Printed Meandering Probe
}

\author{
Ruina Xing ${ }^{1,2}$, Jujin $\mathrm{Li}^{1,2}$ and Dan Sun ${ }^{1,3}$ \\ ${ }^{1}$ AVIC LEIHUA Electronic Technology Research Institute, 214063 Wuxi, Jiangsu, China \\ ${ }^{2}$ Aviation Key Laboratory of Science and Technology on AISSS, 214063 Wuxi, Jiangsu, China \\ ${ }^{3}$ School of Information Science and Technology, Fudan University, 200433 Shanghai, China
}

\begin{abstract}
A broadband probe-fed stacked patch antenna is presented in this paper. The probe is composed of two printed feed-lines and three vias. The feed-lines help of compensating the inductances produced by the vias. Further, with the stacked patch method, the bandwidth of the antenna is enhanced. The simulated results show the antenna has a bandwidth over $40 \%(\mathrm{VSWR}<2)$ and good radiation performance.
\end{abstract}

\section{Introduction}

With the increase of the application of microstrip patch antenna, its bandwidth enhancement has been one of the most attractive research fields. The most convenient method is to use electrically thick substrate. However, in the conventional edge- and probe-fed patch antennas, the method is greatly restricted because of the increasing spurious radiation of the feed-lines and the increasing inductance of the probe resulting from the thick substrate. Hence, in many broadband patch antennas with thick substrates, non-contact feeding method is employed, such as aperture- and proximity-coupling [1-5]. Howbeit, aperture-coupling will increase the back radiation, and the feed structure of proximity-coupling patch antenna will increase the spurious radiation. As compared with non-contact feeding method, direct feeding mechanism is easier to be achieved. In particular, probe-feeding has the potential that the inductance resulting from the probe can be compensated by modifying its structure. Accordingly, the limitation of the substrate thickness is overcome. In literature [6], a meandering probe fabricated by metallic plate was used, and a bandwidth of $24 \%$ was obtained. Further, the bandwidth of the antenna with the feeding approach was improved to $40 \%$ through combining the stacked patch technique [7]. However, the cross-section of the probe formed by the metallic plate is thick due to the requirement for the structure strength, which will influence the matching of antenna in high-frequency band. Furthermore, Furthermore, the metallic plate probe brings on the difficulty of fabrication in high-frequency band applications. For instance, it cannot be manufactured together with the patch by printed circuit technique and the antenna used the air substrate to install the metallic plate probe.

In this paper, a printed meandering probe, instead of the metallic plate probe, is designed to solve the aforementioned problem, and then a broadband stacked patch antenna is designed. With the method, the fabrication difficulty of the antenna is reduced, and its application range is improved to high-frequency band. The bandwidth of the antenna exceeds $40 \%$, and its crosspolarization is less than $-23 \mathrm{~dB}$.

\section{Antenna configuration}

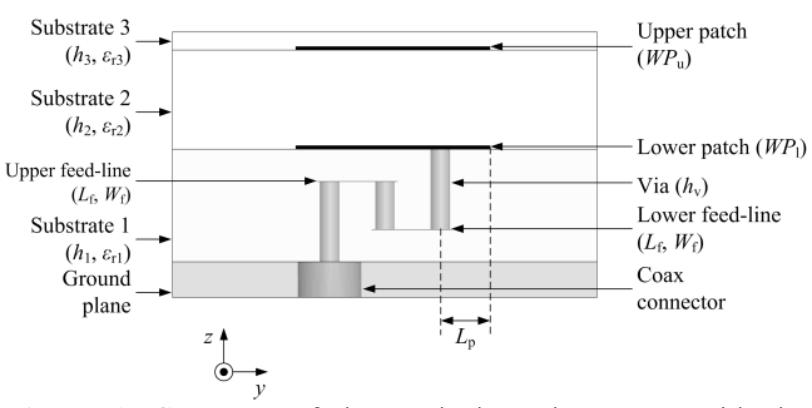

Figure 1. Geometry of the stacked patch antenna with the printed meandering probe.

The antenna fed by the printed meandering probe is shown in Figure 1. The antenna consists of three substrate layers, two stacked patches, a printed meandering probe, and a ground plane. The lower patch is on the upper surface of substrate 1, and the upper patch is located on the lower surface of substrate 3 . The dimensions of the stacked patches are $W P_{1}$ and $W P_{\mathrm{u}}$, respectively. Two feed-lines are located on the different surfaces in substrate 1 , and connected by a via centered with respect to the patch. Each feed-line is symmetrical with respect to the $x$-direction. Its length and width are $L_{\mathrm{f}}$ and $W_{\mathrm{f}}$, respectively. The connection of the lower feed-line and the lower patch, as also the connection of the upper feedline and coax connector, is the same as that of two feedlines. The two vias have the height denote $h_{\mathrm{v}}$. These vias and feed-lines constitute the printed meandering probe. 
The distance between the feeding point of via and the edge of the lower patch is half of $W P_{1}$ minus $L_{\mathrm{f}}$. These vias and feed-lines constitute the printed meandering probe. To fabricate the patch circuits and the vias easily, PTFE with the permittivity of 2.2 is used as the material of substrates 1 and 3 . Their heights are $3.175 \mathrm{~mm}$ and $0.127 \mathrm{~mm}$, respectively. The material of substrates 2 is foam, whose height and permittivity are $3 \mathrm{~mm}$ and 1.27 , respectively.

\section{Antenna design and results}

The two feed-lines of the antenna partly neutralize the inductance produced by the vias, and the good impedance matching is achieved even if the electrically thick substrate is employed in the antenna. Accordingly, the bandwidth of the antenna is increased. To compensate the discontinuity produced by the vertical connection of the feed-lines and the vias, the bonding pads are added at their junctions. The antenna is simulated by HFSS v12. The parameters of the feeding structure and the patches are optimized by the particle swarm optimization (PSO) algorithm [8]. Their solution spacing is shown in Table 1.

Table 1. Range of the optimized parameters (unit: $\mathrm{mm}$ )

\begin{tabular}{|c|c|c|c|c|c|}
\hline Parameters & $W P_{1}$ & $W P_{\mathrm{u}}$ & $h_{\mathrm{v}}$ & $W_{\mathrm{f}}$ & $L_{\mathrm{f}}$ \\
\hline Minimum & 3 & 3 & 1.59 & 0.3 & 0.4 \\
\hline Maximum & 12 & 12 & 3 & 2.5 & $W P_{\mathrm{l}} / 2-0.8$ \\
\hline
\end{tabular}

The numbers of the particles are ten, and the fitness function is defined as (1).

$$
\begin{aligned}
f(x)= & \left(\left|\operatorname{VSWR}_{8 \mathrm{GHz}}-1.5\right|+\left|\mathrm{VSWR}_{12 \mathrm{GHz}}-1.5\right|\right) / 2 \\
& +\max \left(\mathrm{VSWR}_{9 \mathrm{GHz}-11 \mathrm{GHz}}\right)-1
\end{aligned}
$$

With the optimization of parameters, a designed instance is exhibited. Its designed parameters are as follows: $W P_{1}=8.37 \mathrm{~mm}, \quad W P_{\mathrm{u}}=8.4 \mathrm{~mm}, \quad W_{\mathrm{f}}=2.16 \mathrm{~mm}$, $L_{\mathrm{f}}=1.735 \mathrm{~mm}, h_{\mathrm{v}}=2.26 \mathrm{~mm}$. The simulated bandwidth of

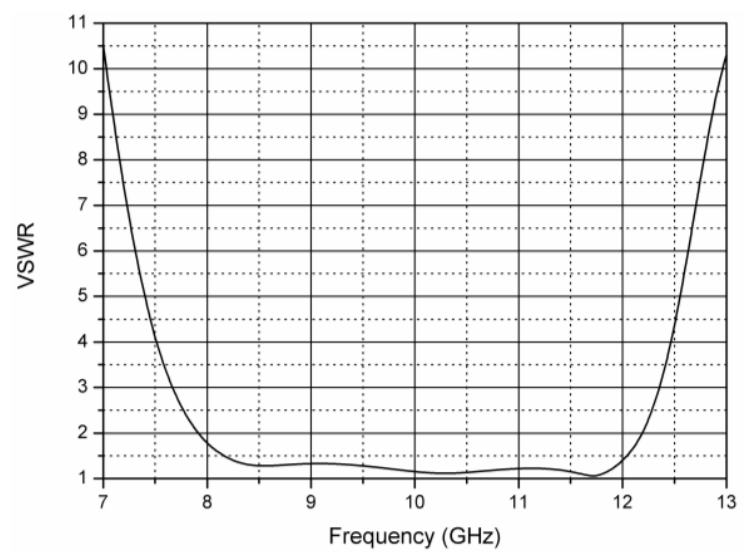

Figure 2. Simulated VSWR of the antenna. the antenna is revealed in Figure 2. As can be seen, the operation band with VSWR below 2 is from $7.92 \mathrm{GHz}$ to $12.18 \mathrm{GHz}$, and its relative bandwidth is $42.4 \%$. Furthermore, in the frequency range of $8.17 \mathrm{GHz}$ to $12.04 \mathrm{GHz}(38.3 \%)$, the VSWRs are all lower than 1.5, and the ripples of the VSWR curve are small across the entire impedance band. The bandwidth simulated result indicates that the antenna with the printed meandering probe have a good broadband response which is evenly matched with that of the antenna in [7], when retaining the advantage of easy fabrication.

Figure 3 shows the radiation patterns of the antenna. From the simulated results, it can be seen that the good polarization purity is achieved. The cross-polarization

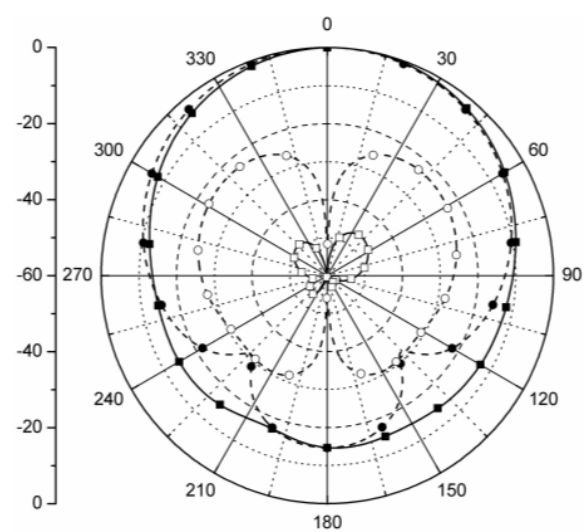

(a)

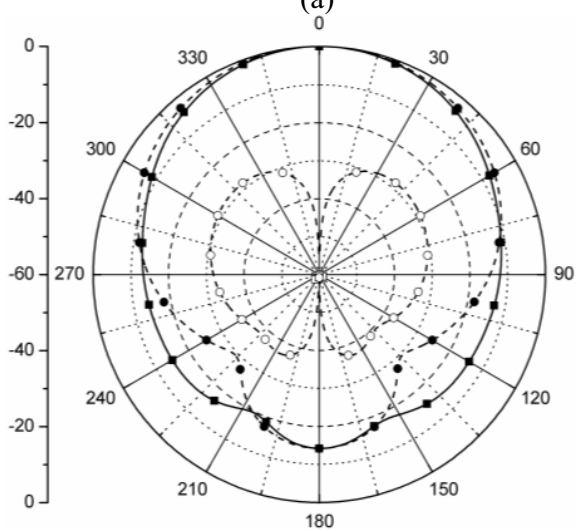

(b)

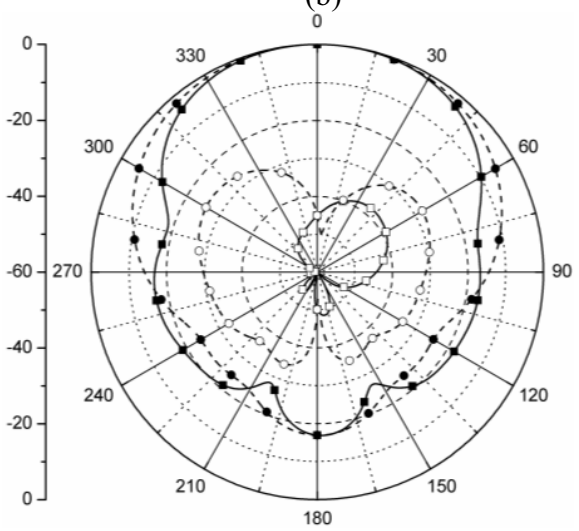

(c)

Figure 3. Simulated radiation patterns at different frequencies. (a) $8 \mathrm{GHz}$; (b) $10 \mathrm{GHz}$; (c) $12 \mathrm{GHz}$; $\rightarrow$ Co-pol (E plane); Co-pol (H-plane); $-\square-$ Cross-pol (E-plane); - - Cross-pol (H-plane) 
levels in $\mathrm{E}$ and $\mathrm{H}$ planes are less than $-38 \mathrm{~dB}$ and $-23 \mathrm{~dB}$, respectively. And at the frequency of $10 \mathrm{GHz}$, its values are lower than $-58 \mathrm{~dB}$ and $-28 \mathrm{~dB}$, respectively. With the increase of frequency, the spurious radiation of the feedlines increases. This results in a small asymmetry of the radiation pattern in $\mathrm{E}$ plane at $12 \mathrm{GHz}$. In the meantime, the cross-polarization is deteriorated. Because the ground plane with the dimensions of $13.3 \mathrm{~mm} \times 13.3 \mathrm{~mm}$ is small, the front-to-back ratio is not high, whose value is only better than $14 \mathrm{~dB}$. But it can be improved simply by increasing the ground's sizes. On the whole, the antenna shows the pretty radiation performance in the operation band.

\section{Feed-line and patch analyses}

As is stated above, the matching performance of the antenna is influenced by the thickness of the cross-section of the feeding structure. If the feed-line is thick, its matching will be deteriorated rapidly. Figure 4 reveal VSWRs of the antenna with the different thickness of the feed-line. While its thickness is $0.018 \mathrm{~mm}$ (typical value of the copper claddings on laminates), the matching performance is almost the same as that of the ideal condition (thickness $=0 \mathrm{~mm}$ ). Also, this means the PTFE materials and the printed circuit technique will not influence the performance of the antenna. For ensuring the enough structure strength, the thickness of the feedline composed of metallic plate is at least $0.1 \mathrm{~mm}$, and it reaches $1 \mathrm{~mm}$ in most case. As can be seen in Figure 4, when its thickness is $0.1 \mathrm{~mm}$, the VSWRs of the antenna are increased, which means the matching performance is worsened. Furthermore, the degree of the deterioration will be serious when the thickness is more than $0.3 \mathrm{~mm}$. The phenomenon is caused by the mismatching of the resonant impedances. Figure 5 shows the impedance of the antenna with different thickness of the feed-line. Because the two staked patches produce two resonances, there are two peak values in the resistance curves seen from Figure 5(a). As the feed-line is thin (i.e. the curves of $0 \mathrm{~mm}$ and $0.018 \mathrm{~mm}$ ), the resistances of the antenna are approach to $50-\Omega$ in the operation band. In the meantime, the reactance of the antenna is close to zero. With the enhancement of the thickness, the resistances are

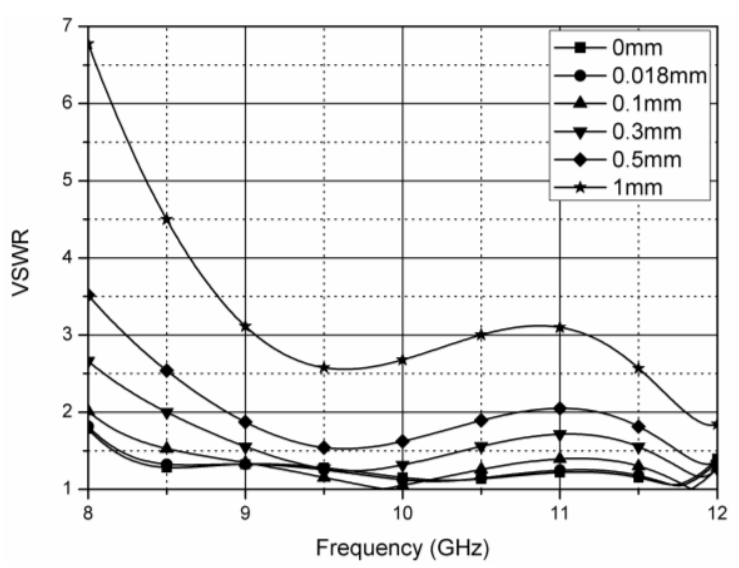

Figure 4. VSWR versus thickness of the feed-line. increased. In particular, the resistances nearby the upper resonance are shot up. In addition, the range of reactance variation is increased extremely. These characteristics means the impedances of the antenna is mismatch $50-\Omega$ coax connector.

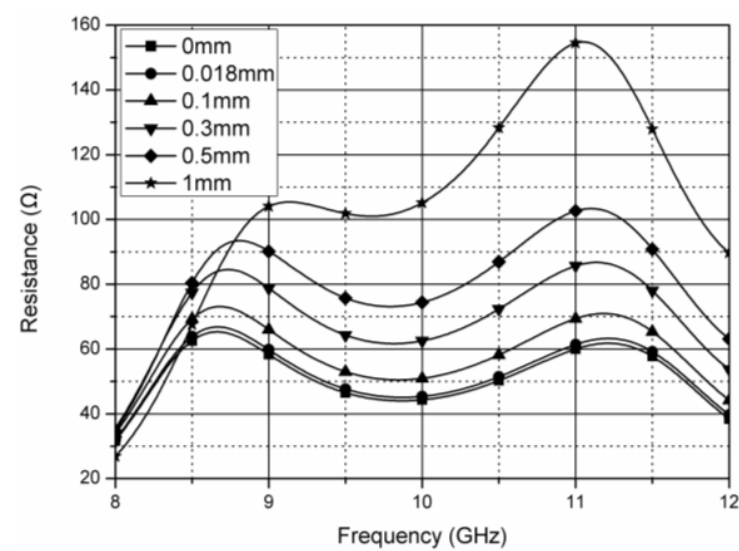

(a)

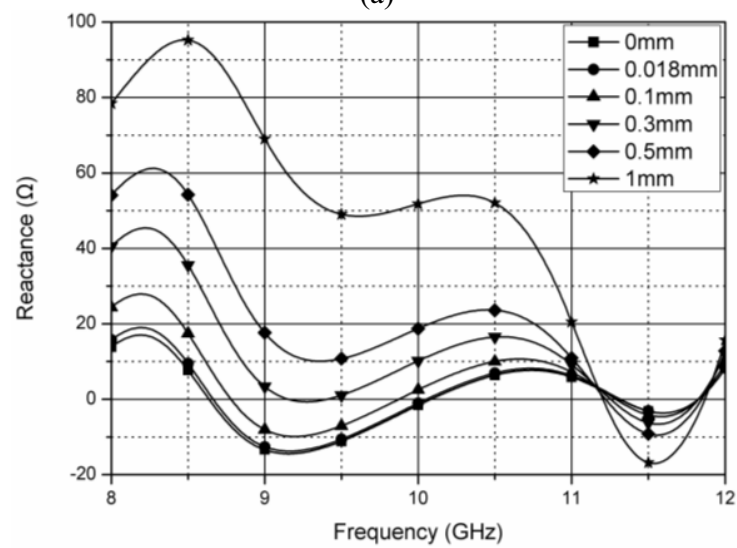

(b)

Figure 5. Input impedance versus thickness of the feed-line. (a) resistance; (b) reactance

The feeding structure influences not only the matching performance, but also the polarization purity. Figure 6 gives the maximum cross polarization of the antenna with different thickness of the feed-line. As compared with the effect of the feed-line on the matching

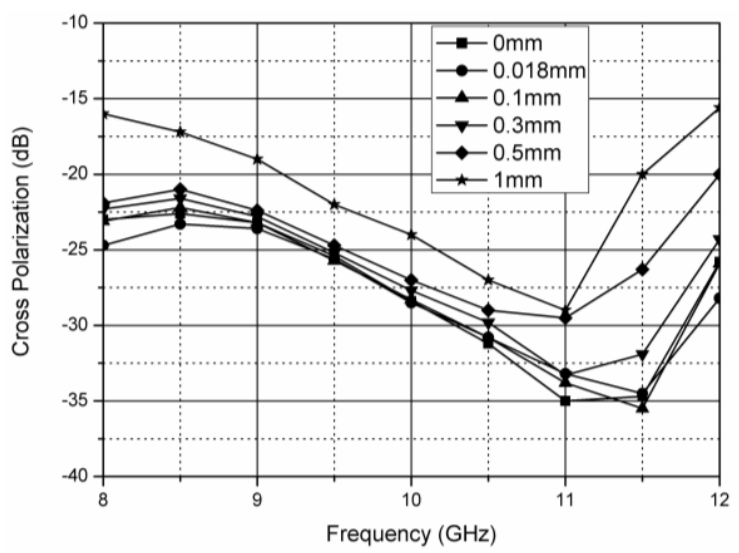

Figure 6. Maximum cross polarization level versus thickness of the feed-line. 
performance, that on the cross polarization is significantly smaller with the thickness below $0.5 \mathrm{~mm}$. However, with the further increase of the thickness, the polarization purity degenerates appreciably. While its thickness equals $1 \mathrm{~mm}$, the cross polarization level is more than that of ideal condition by the maximum value of $14.7 \mathrm{~dB}$. The foregoing depictions indicate the feeding structure made up of the metallic plate is unfavorable for the application in high-frequency band.

The enlargement of bandwidth depends mainly on the stacked patch besides the feeding structure. The two patches affect the resonant resistance and frequency. Figure 7 demonstrates the impedance variations of different $W P_{1}$. As can be seen form Figure 7(a), the lower resonant resistance is improved with the increases of $W P_{1}$. Meanwhile, the frequency of the lower resonance is shifted downward to the lower-frequency band. As compared with the changes of the lower resonance, those of the upper resonance are relatively smaller. Its resistance and frequency are varied smoothly. With the further decrease relative to the optimum design, they remain stable basically. This means the lower patch dominates mostly the lower resonance.

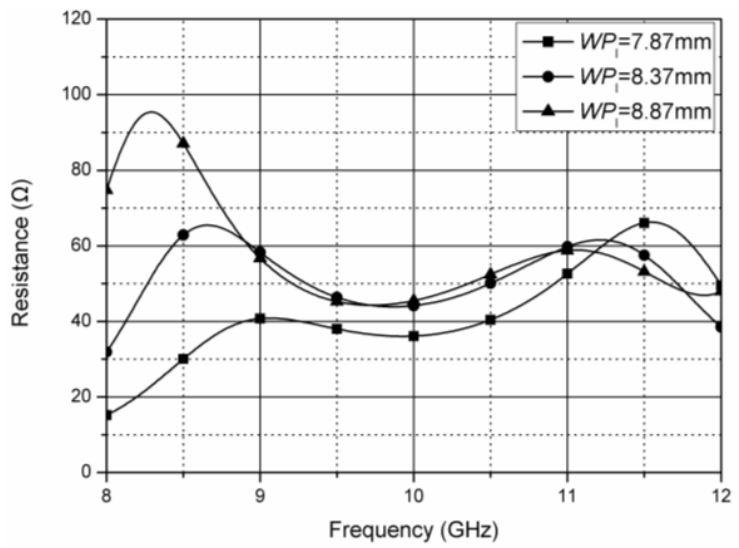

(a)

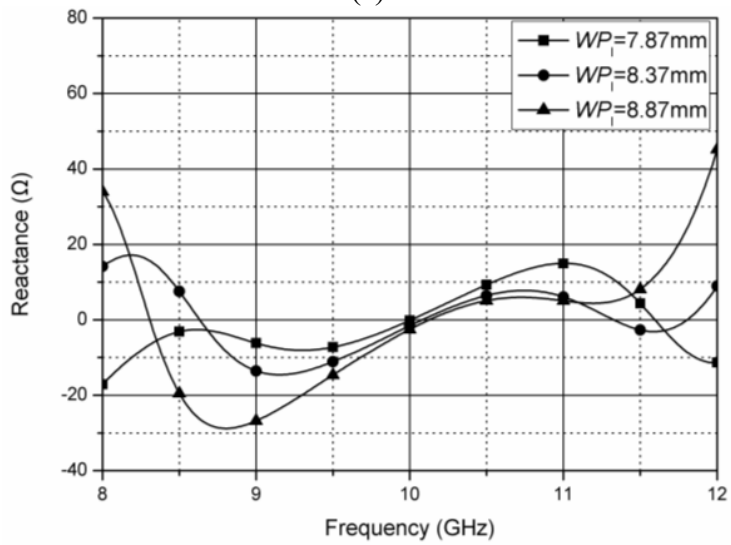

(b)

Figure 7. Input impedance versus $W P_{1}$. (a) resistance; (b) reactance

The effects on the impedance of different $W P_{\mathrm{u}}$ are shown in Figure 8. As $W P_{\mathrm{u}}$ increases, the lower resonant resistance is reduced, which is opposite to the trend with the changes of $W P_{1}$. This is because its extension means the lower patch size relative to the upper patch size decreases. It also can be seen that the increase of $W P_{\mathrm{u}}$ make the upper resonant frequency be shift downward to the lower-frequency band quite a bit, but the lower resonant frequency is almost unchanged. Also, the augment of $W P_{\mathrm{u}}$ results in increasing the upper resonant resistance. These behaviors indicate the upper resonance is influenced mainly by the upper patch. Actually, due to the coupling between the two patches, both of the two resonances are affected by the variation of each of them, which can be seen in Figures 7 and 8. The difference is the degree of their influences. On the whole, the large frequency interval and the small impedance alteration range between the two resonances contributes to improvement of the antenna bandwidth, and the balance of the two resonant impedances is prerequisite for achieving this goal.

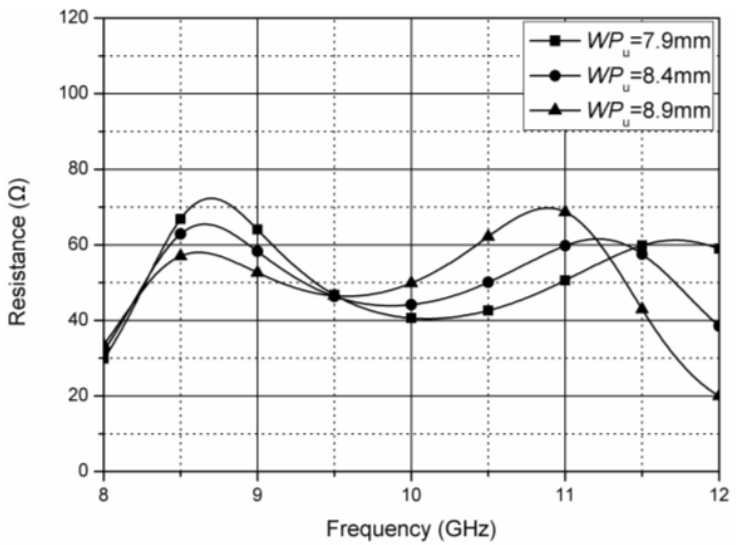

(a)

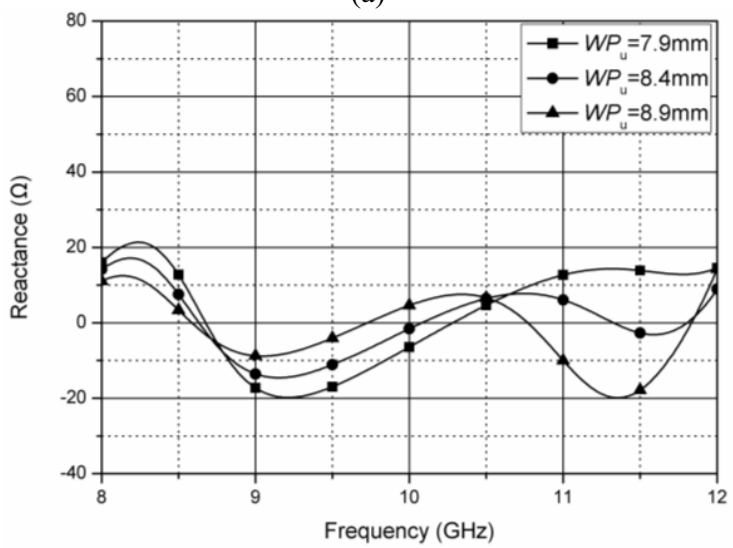

(b)

Figure 8. Input impedance versus $W P_{\mathrm{u}}$. (a) resistance; (b) reactance

\section{Conclusion}

A wideband staked patch antenna fed by a printed meandering probe has been designed in this paper. The feeding structure helps in the broadband impedance matching of the antenna, and has the feature that easy of manufacture. The excellent simulated results of the antenna show the broadband over $40 \%(\mathrm{VSWR}<2)$ and the cross-polarization below $-23 \mathrm{~dB}$, which demonstrates that the method is effective. Furthermore, due to the 
simple and compact structure of the antenna, it is suitable for assembling an array.

\section{References}

1. S. D. Targonski, R. B. Waterhouse, D. M. Pozar, Design of wide-band aperture-stacked patch microstrip antennas, IEEE Trans. Antennas Propagat. 46, 9, pp. 1245-1251 (1998)

2. Y. Qin, S. Gao, A. Sambell, E. Korolkiewicz, M. Elsdon, Broadband patch antenna with ring slot coupling, Electron. Lett. 40, 1, pp. 5-6 (2004)

3. K. Oh, B. Kim, J. Choi, Design of dual and wideband aperture-stacked patch antenna with double-sided notches, Electron. Lett. 40, 11, pp. 643644 (2004)

4. I. Ang, B. L. Ooi, Broadband semi-circle-fed microstrip patch antennas, IET Microw. Antennas Propagat. 1, 3, pp. 770-775 (2007)

5. D. Sun, L. You, A broadband impedance matching method for proximity-coupled microstrip antenna, IEEE Trans. Antennas Propagat. 58, 4, pp. 13921397 (2010)

6. H. W. Lai, K. M. Luk, Wideband patch antenna with low cross-polarisation, Electron. Lett. 40, 3, pp. 159160 (2004)

7. H. W. Lai, K. M. Luk, Wideband Stacked patch antenna fed by meandering probe, Electron. Lett. 41, 6, pp. 297-298 (2005)

8. S. Xu, Y. Rahmat-Samii, Boundary conditions in particle swarm optimization revisited, IEEE Trans. Antennas Propagat. 55, 3, pp. 760-765 ( 2007) 